\title{
Lightweight Process Support with Spreadsheet-Driven Processes: A Case Study in the Finance Domain
}

\author{
Michael Stach ${ }^{1}$, Rüdiger Pryss ${ }^{1}$, Maximilian Schnitzlein ${ }^{1}$, Tim Mohring ${ }^{1}$, \\ Martin Jurisch ${ }^{2}$, Manfred Reichert ${ }^{1}$ \\ ${ }^{1}$ Institute of Databases and Information Systems, Ulm University, Germany \\ http://www. uni-ulm.de/dbis \\ ${ }^{2}$ AristaFlow GmbH, Germany \\ http://www.aristaflow.com \\ \{michael.stach, ruediger.pryss, maximilian.schnitzlein, tim.mohring, \\ manfred.reichert\}@uni-ulm.de \\ martin.jurisch@aristaflow.com
}

\begin{abstract}
The use of process management technology constitutes a salient factor for a multitude of business domains as it particularly addresses the flexibility demands of the digital enterprise. Still, spreadsheet applications are more likely to be used in many scenarios in which process management technology appears to be a more appropriate solution. Especially in the context of human-centric and knowledge-intensive processes, spreadsheets are widely used, even if more business-tailored applications exist. For example, financial service providers, like banks or insurers, prefer spreadsheet applications for accomplishing their daily business. However, this kind of usage reveals drawbacks when working collaboratively based on the same spreadsheet document. To remedy these drawbacks, we suggest the use of spreadsheet-driven processes, which shall combine the advantages of traditional process management technology with the ones of spreadsheets. Using a sophisticated scenario from the financial domain, this paper shows how spreadsheet-driven processes improve collaborative work, as required in the context of business processes, significantly. Moreover, a proof-of-concept prototype is presented to evaluate the approach in practice. Altogether, first results indicate that spreadsheet-driven processes may be a promising technical solution for everyday business involving human resources.
\end{abstract}

Keywords: human-centric, spreadsheet-driven process, financial field

\section{Introduction}

During the last decade, the support of human-centric and knowledge-intensive processes has gained increasing attention [11]. In general, corresponding processes face unplanned and complex situations, and their enactments depends on the expertises and skills of the stakeholders involved. In particular, an unmanageable set of factors makes it impossible for process stakeholders to always 
foresee all details of the tasks they shall perform and coordinate with other stakeholders. Particularly, these processes comprise mutual phases of planning work and performing it. Usually, in these phases, spreadsheet documents are used to support the respective planning and working tasks. In addition, spreadsheet documents are used to coordinate the tasks of the collaborating stakeholders. Human-centric, knowledge-intensive processes of this kind can be found in domains like, for example, healthcare or finance [5].

Due to regulatory changes in the financial field [12], recently, for many service providers (e.g., banks), the support of human-centric, knowledge-intensive processes is increasingly demanded; e.g., the risk reporting procedure provides a scenario in which recent regulatory changes have generated high demands for any IT solution. More precisely, the management of human resources is particularly challenging. For example, many decisions concerned with the evaluation of risks must be accomplished by more than two experts. To coordinate such decision making process with the help of IT solutions, in turn, often requires a costly adaptation of existing software systems.

In a case study that we conducted in a bank, we learned that risk reporting procedures are still mainly managed based on spreadsheet documents. Regarding the considered bank in particular, and financial service providers in general, the use of spreadsheet documents poses three major advantages:

- Spreadsheet documents are easy to manage, intuitive, and well established.

- Spreadsheet documents can be easily extended.

- Spreadsheet documents provide powerful off-the-shelf features (e.g., calculation functions, chart-based data visualization).

The large-scale use of spreadsheet documents, however, reveals many drawbacks. For example, it is a challenging endeavor for all collaborating stakeholders to work on the same spreadsheet document concurrently without generating unintended side effects (e.g., a corrupt document state). More specifically, avoiding undesired side effects must be accomplished manually, which, in turn, is an errorprone task. To remedy this drawback, while still using the well-known spreadsheet metaphor, we propose spreadsheet-driven processes combining spreadsheet with process management technology. Conceptionally, spreadsheet-driven processes aim to preserve the aforementioned advantages of spreadsheet documents on one hand, while avoiding unintended side effects in the context of collaborative work on one and the same spreadsheet document on the other. Technically, spreadsheet-driven processes are enabled by enhancing existing process management technology in two respects. First, we tightly integrate spreadsheet applications with a robust process engine. Second, we adjust the data handling concept used by common process engines in order to integrate spreadsheet documents for handling data.

In the aforementioned case study, we presented the concept of spreadsheetdriven processes to the stakeholders involved in the risk reporting procedure. As our basic ideas were rated very positively, we realized a proof-of-concept prototype enabling spreadsheet-driven processes based on the AristaFlow Business 
Process Management Suite [2]. This paper reports on three aspects. First, we present the case study, and second, we introduce the concept of spreadsheetdriven processes, and third, we give insights into the proof-of-concept prototype.

The remainder of the paper is structured as follows: Section 2 introduces the risk management procedure, the reporting procedure as well as the risk-bearing capacity procedure in detail. Note that this procedure constitutes a fundamental part of the overall risk management procedure. Section 3 then introduces the concept of spreadsheet-driven processes. The proof-of-concept prototype is presented in Section 4. Finally, Section 5 discusses related work and Section 6 concludes the paper with a summary and outlook.

\section{Risk Management Procedure}

This section presents the overall risk management procedure in general as well as its risk reporting part in particular. Note that the risk-bearing capacity procedure this work is exemplarily focusing on constitutes one crucial part of the risk reporting. Fig. 1 illustrates the risk management procedure. In a nutshell, it consists of two parts. The first one is risk inventory, which comprise the strategical tasks, whereas the second part is risk management, which comprises the operational tasks. In the context of risk inventory, in turn, risks (e.g., deficits) are identified and rated, while in the context of risk management they will be handled. To be more precise, the risk inventory part consists of activities risk detection and risk rating. In turn, risk measurement, risk reporting, risk controlling, and risk monitoring constitute the activities of the risk management procedure. At first glance, the risk management procedure seems to be of poor complexity. In practice, however, its handling is challenging as the involved actors need to be coordinated. For example, for almost all activities to be performed, a binding of duties becomes necessary. Thereby, a binding of duty entailment constraint expresses that two independent persons shall approve an activity.

RISK INVENTORY

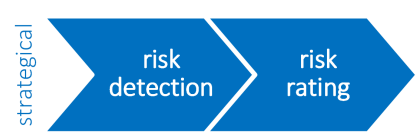

RISK MANAGEMENT

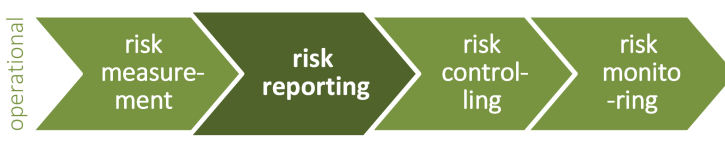

Fig. 1. Risk Management Procedure

\subsection{Risk Reporting Procedure}

To give an idea of the complexity of risk management [10], Fig. 2 illustrates major parts of the risk reporting procedure. First, overall risk reporting is accomplished quarterly. Second, it comprises five sub-procedures: management summary, risk 
metrics, risk-bearing capacity, single risks, and issue management. Ideally, the five sub-procedures can be executed in parallel in order to minimize overall execution time. After completing all sub-procedures, a second phase of the risk reporting procedure, consisting of seven activities, needs to be coordinated. This second phase starts with the assembly of a preliminary version of the risk report (1). For this purpose, documents created by the mentioned sub-procedures are collected and compiled to a single document (2), i.e., the risk report. Using the compiled report, a consultation with the risk controller takes place (3). As an outcome of this consultation, the risk controller decides whether or not adjustments to the risk report become necessary. If adjustments are required, activity include additions is performed (4). If no or no more adjustments are needed, the risk report must be discussed with a responsible board member (5). Again, it needs to be decided whether additional adjustments are required (6). After the risk report is approved by the responsible board member, it is presented to the executive board (7). The resulting risk report is then dispatched to a specified list of addressees (8). Note that Fig. 2 only reflects a simplified version of the risk reporting procedure. In daily business, there are many other interdependencies to be considered. Nevertheless, the presented risk reporting procedure provides a proper basis for discussing the risk-bearing capacity procedure.

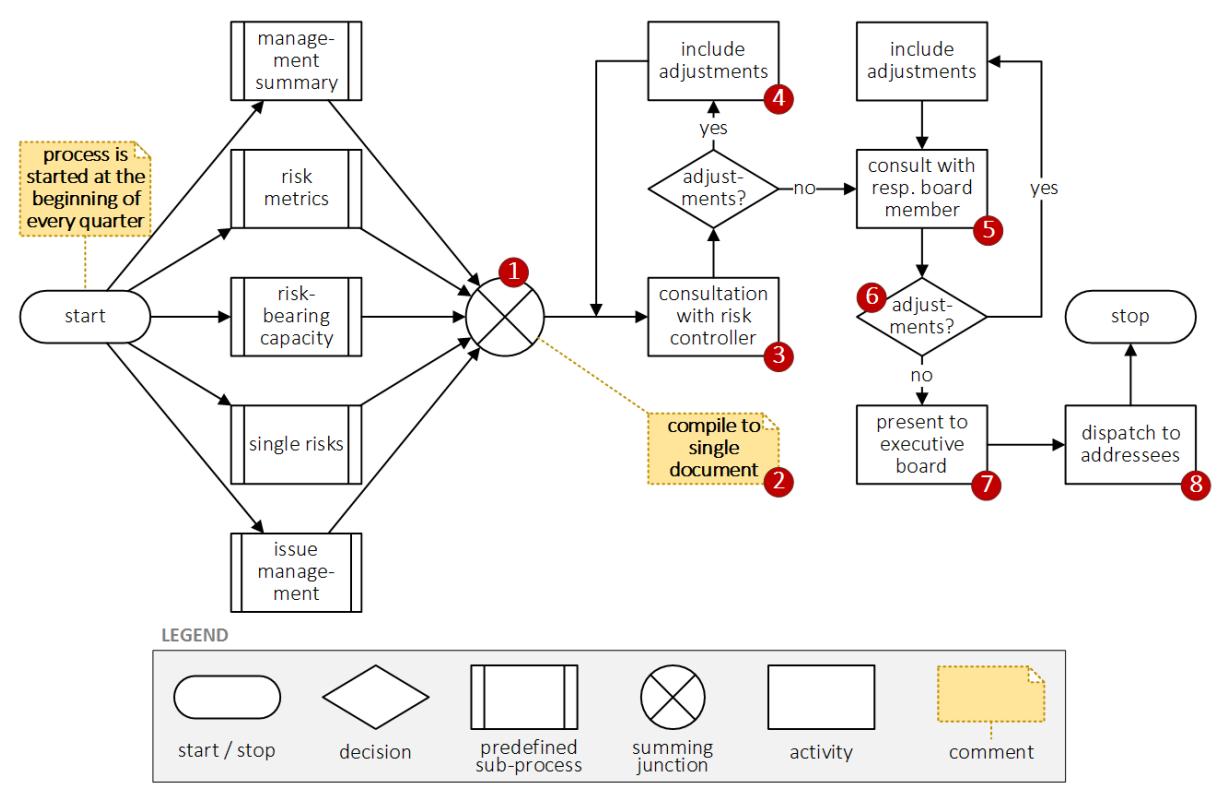

Fig. 2. Risk Reporting Procedure (in Flowchart Notation) 


\subsection{Risk-bearing Capacity Procedure}

In the context of the aforementioned case study, we learned that the risk-bearing capacity procedure (RBCP) [6] constitutes the one of the five risk reporting subprocedures for which the coordination efforts between the involved actors might be significantly decreased when providing a proper IT support. Based on Fig. 3 , we illustrate the specific aspects of the RBCP that require sophisticated IT support not existing so far:

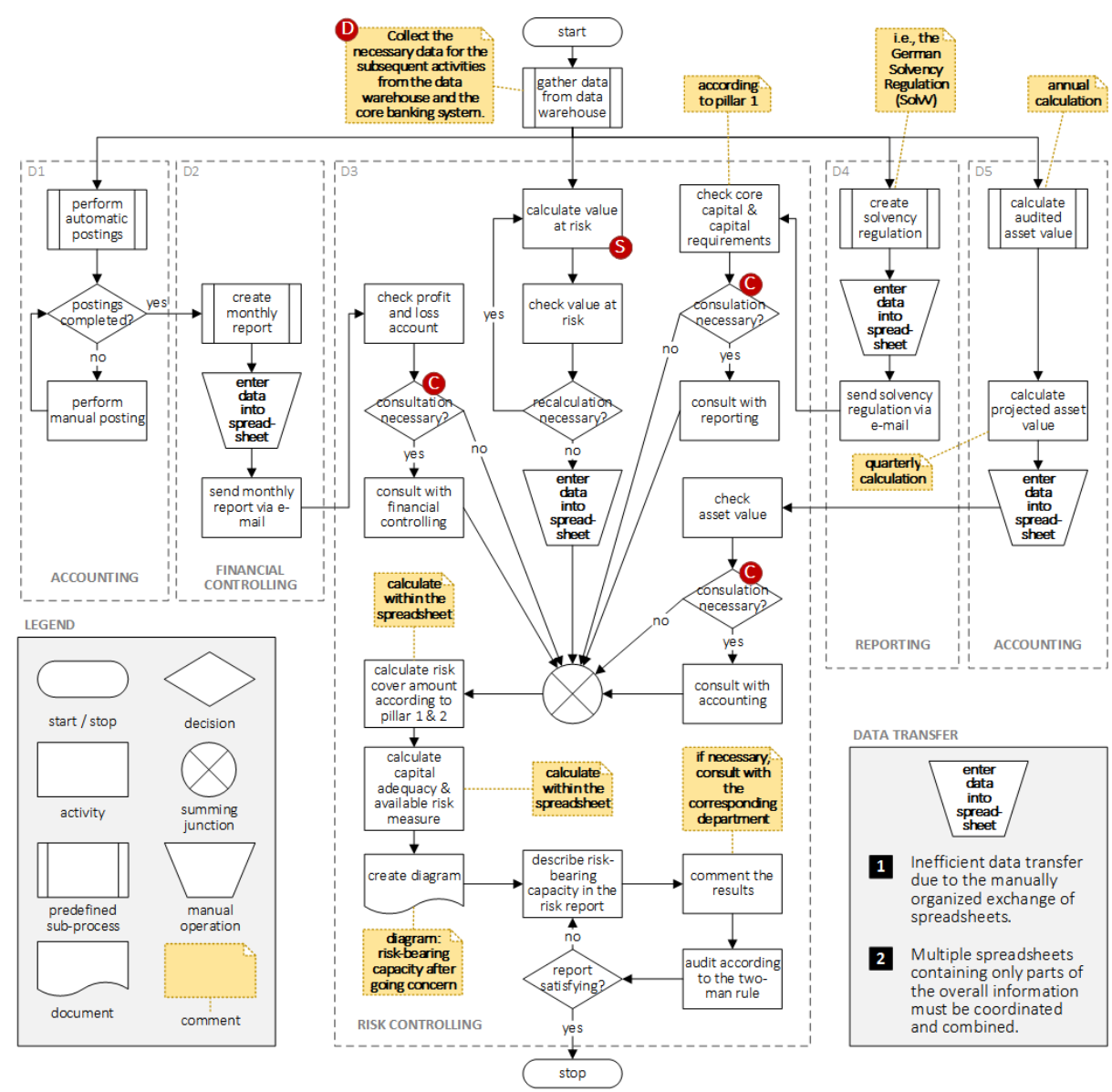

Fig. 3. Risk-bearing Capacity Procedure (in Flowchart Notation)

- In total, the activities from five different departments (cf. Fig. 3, D1-D5) need to be coordinated, i.e., accounting, financial controlling, risk controlling, reporting, and accounting of leasing company. Note that the involvement of 
the leasing company requires the consideration of different organizational scopes.

- RBCP comprises challenging data collection tasks (cf. Fig. 3, (D)) involving numerous heterogeneous IT systems. Several of these tasks, in turn, require the use of simulation tools (cf. Fig. 3, S). As a drawback, currently, simulation results need to be manually transferred to the relevant spreadsheet.

- Several RBCP parts show variations in practice. In Fig. 3, they are represented as sub-procedure activities (e.g., create monthly report).

- Data, exchanged between departments, needs to be approved (cf. Fig. 3, C)), necessitating costly consultation activities.

- In the current practice, spreadsheets are used to coordinate data exchange activities between the stakeholders involved in the RBCP. In turn, this results in spreadsheet-related drawbacks SRD1-SRD5:

SRD1 Spreadsheet management (i.e., to send, receive, store spreadsheets) is manually organized through e-mails, file sharing, or phone calls.

SRD2 A sophisticated rights management for the use of spreadsheet documents is difficult to realize.

SRD3 Manual spreadsheet management hampers fine-grained rights management that allows distinguishing rights regarding the different parts of a spreadsheet document. In practice, such a fine-grained rights management would be highly welcome in the RBCP context.

SRD4 Though spreadsheets constitute the primary data handling instrument, manual data transfer between the spreadsheets and other systems (e.g., the simulation tool used for activity calculate value at risk) is often required.

SRD5 Recall that the risk reporting procedure, which includes RBCP as sub-procedure, is executed quarterly (cf. Fig. 2). In practice, the order of the execution activities and the used spreadsheets (i.e., how many spreadsheets are actually used) of the risk reporting procedure vary quarterly. Therefore, users want to operate with the same and only one spreadsheet document. To cope with multi-user scenarios in the context of single spreadsheets, a feature must be provided that enables a parallel use in this context. Such feature, however, cannot be easily realized using off-the-shelf spreadsheet applications.

\section{Towards Spreadsheet-Driven Processes}

Section 2 has confirmed that the RBCP requires considerable coordination efforts and involves many stakeholders. Furthermore, RBCP activities are mostly executed in the same sequence each quarter of the year. Considering these two issues, the use of flexible process management technology for the RBCP is promising [14]. Even the use of contemporary process management technology for the support of risk management procedures in general, and the RBCP in particular, however, has revealed several drawbacks as reported by the stakeholders during our case study. To obtain deeper insights into these drawbacks, we consider Fig. 
4. Assume the following scenario: The RBCP shall be executed by a process engine [15]. Then, additional information is required to transform the RBCP to an executable process. For example, data elements need to be defined and assigned to the activities reading or writing them. In current practice, lots of data from different sources need to be handled. Consider the data warehouse rectangle shown in Fig. 4. It illustrates that the different sources to be handled lead to a massive definition of data elements and their assignments to activities. Furthermore, for each created data element, the rights of the involved stakeholders, who may operate on these data elements, need to be defined. On one hand, the use of process management technology allows for a technically sound representation of the RBCP. On the other, the maintenance of the aforementioned required data elements is still complex. Taking this into account, we propose to combine advanced process management technology with spreadsheets (cf. Fig. 4). This integration follows three goals:

1. It addresses the discussed spreadsheet-related drawbacks of the current processing of the RBCP (cf. Section 2.2). Concerning SRD1, for example, the spreadsheet management is automated.

2. It copes with the data handling drawbacks discussed in this section, i.e., it addresses the scenario depicted in Fig. 4, (1).

3. It allows users to continue working in the spreadsheet environment.

Considering these goals, the approach applies the following concept:

- Data entry as well as data retrieval are performed using spreadsheets.

- The spreadsheet management (i.e., to send, receive, store spreadsheets) is governed through a well-defined process as specified for the RBCP.

- Data handling of any supported business process is completely managed through one and the same spreadsheet.

From a technical perspective, the following adaptations are required when integrating existing process management technology with spreadsheets:

1. All activities reading or writing data are assigned to a spreadsheet document (cf. Fig. 4, (2)). Note that this requires an enhancement of the modeling component as the structure of the spreadsheet must be obtained automatically.

2. The process engine executing a process needs to be adjusted for the use of spreadsheets as well. In this context, three further adaptations are required:

(a) The process engine needs to be tightly integrated with a spreadsheet application (e.g., Microsoft Excel). For example, the process engine needs to be able to automatically run the spreadsheet application.

(b) The process engine needs to be able to use spreadsheets for data handling as illustrated in Fig. 4, (2).

(c) The process engine should ensure that the spreadsheet (1) shall be automatically provided solely to the assigned editor (i.e., the user processing the spreadsheet). Further, it needs to guarantee that the spreadsheet (2) never runs into a corrupt state (e.g., through a multi-user access). Finally, (3) a sophisticated rights management (e.g., cell protection) needs to be implemented. 
1 PROCESS APPROACH

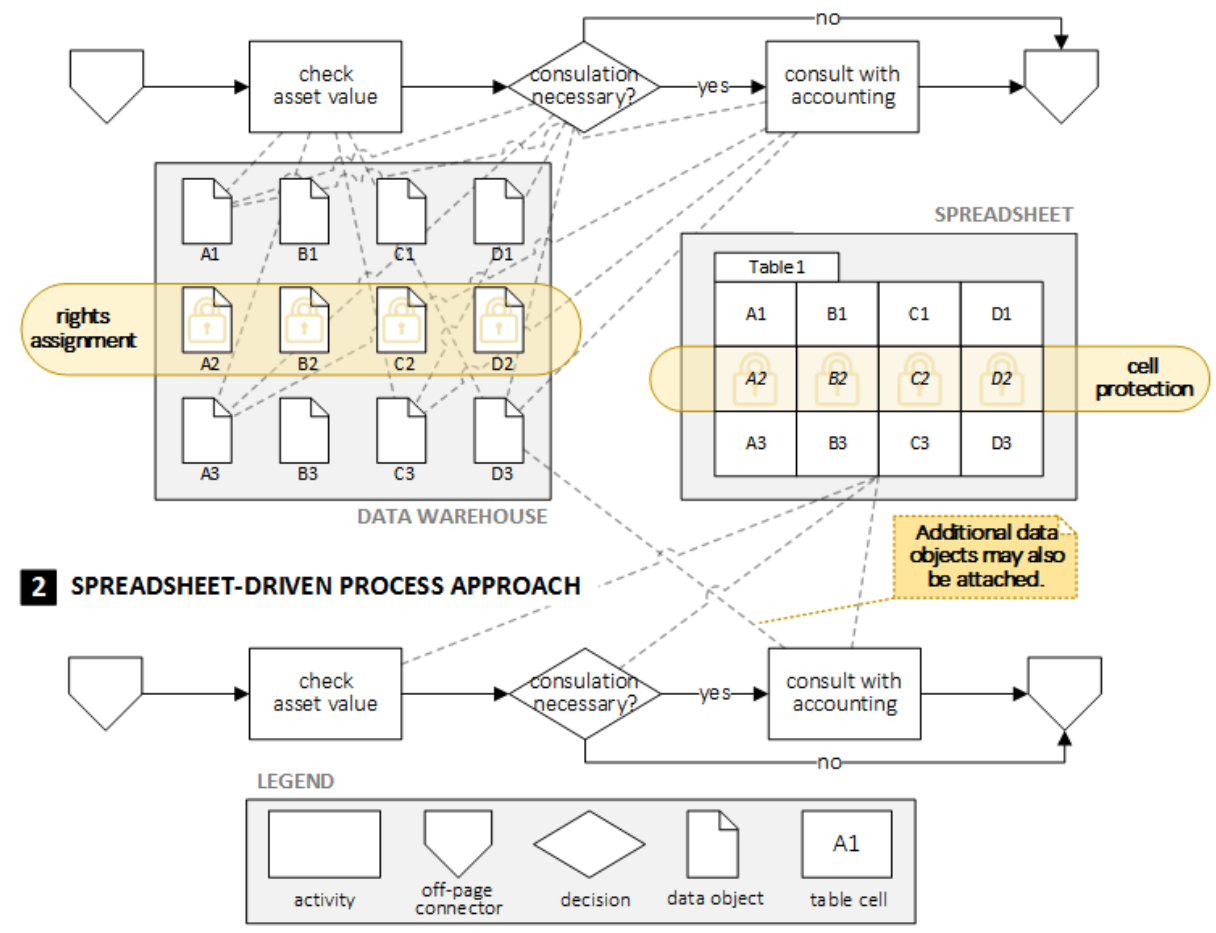

Fig. 4. Data Elements in Spreadsheet-driven Processes (in Flowchart Notation)

When following such an approach, several advantages emerge:

1. An editor has read access to the entire spreadsheet while performing any activity. In particular, every editor can obtain all data already entered by other editors.

2. When processing an assigned activity, an editor may implicitly obtain the current process status (e.g., through data that has not been entered yet, but still has to be provided).

3. When processing an assigned activity, the spreadsheet application may comprise additional and useful features not provided by the process engine. For example, the spreadsheet application can be used to visualize data based on the supported charts.

In summary, we aim at adopting modern process management technology to enable Scenario 2 (cf. Fig. 4) as replacement of Scenario 1 (cf. Fig. 4). We denote processes executed according to Scenario 2 as Spreadsheet-Driven Processes. The mentioned case study revealed that the latter will foster RBCP support in particular and risk reporting in general. 


\section{Proof-of-Concept Prototype}

This section illustrates the proof-of-concept prototype we implemented in order to enable the Spreadsheet-Driven Process as such as the one supporting the RBCP. The prototype was realized with Spreadsheet Router, a software toolset recently released by the AristaFlow Inc.. The toolset, in turn, is based on the AristaFlow Business Process Management Suite [2]. Due to the lack of space, only selected features of the proof-of-concept prototype are presented. Figs. 5 and 6 show selected screens of Spreadsheet Router. Fig. 5 illustrates a screen during the creation of the spreadsheet-driven process supporting the RBCP, while Fig. 6 illustrates a screen when executing the created process. Specifically, Fig. 5 shows one of the three configuration steps required to create the spreadsheetdriven process for the RBCP. To be more precise, the screen shows:

(1) A structured activity list: Based on this list, process activities may be created. In addition, within this screen one may obtain the entire process view of the modeled activities (see bottom of Fig. 5).

(2) Spreadsheet area: Using this area, spreadsheet parts (i.e., so-called spreadsheet areas) of the document can be assigned to the process activities.

(3) Resource assignment: Using this area, it is specified which editors get the right (e.g., specifying roles) to perform a particular process activity; i.e., getting the right to access an area of the spreadsheet in the context of the specified process activity.

Fig. 6 depicts a screen during the execution of the RBCP process. In particular, it shows a form for the activity check core capital $\&$ capital requirements (cf.

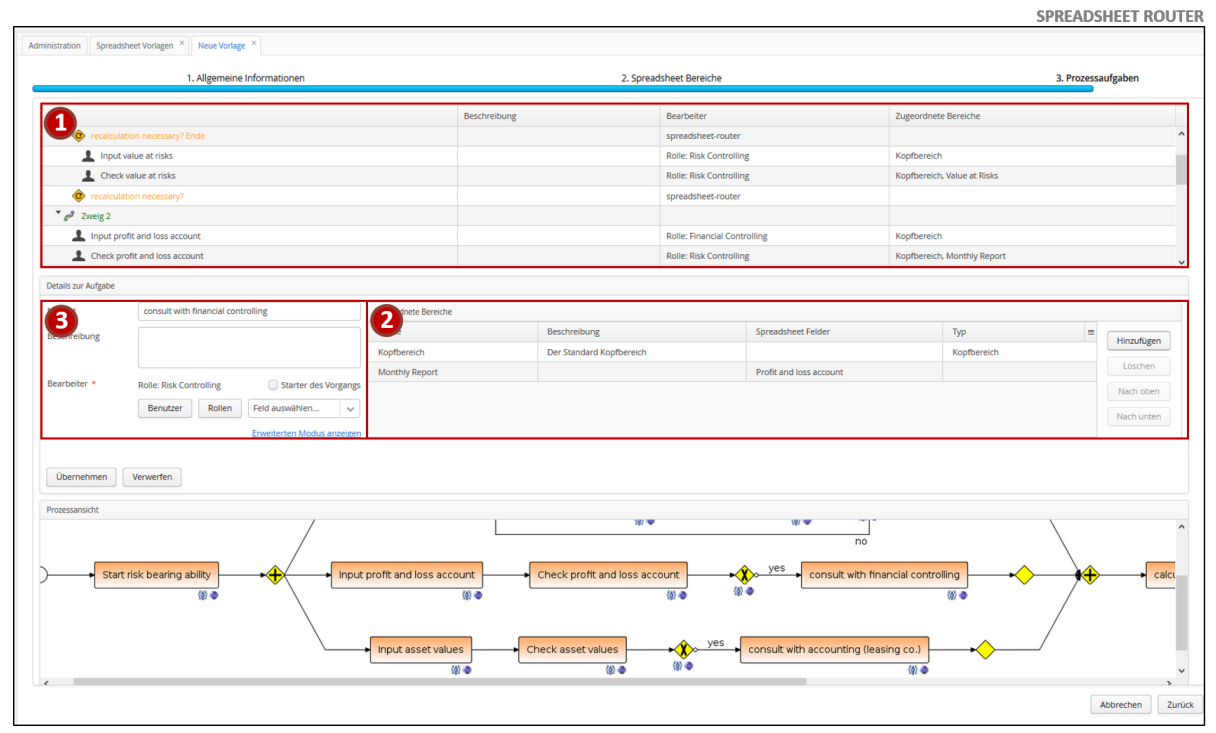

Fig. 5. Spreadsheet-driven Process Creation 


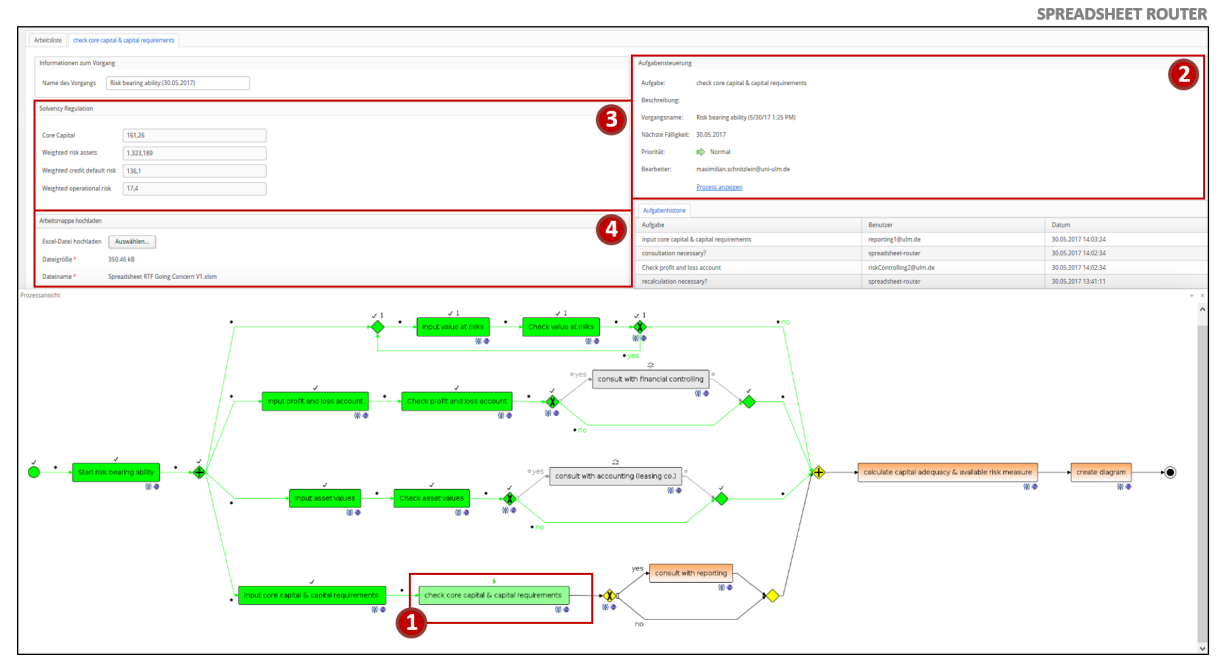

Fig. 6. Spreadsheet-driven Process Execution

Fig. 6, (1), (2)). Currently, this activity was processed by an editor through the use of the spreadsheet application. If the editor wants to again change already processed data, he or she must start the spreadsheet application again with the button shown in Fig. 6 at position (4). Note that in the presented case the spreadsheet area consists of four table cells (cf. Fig. 6, (3)). Furthermore, Spreadsheet Router provides a process-oriented view, e.g., to monitor the current execution status of the process (see the bottom of Fig. 6).

Overall, we realized a proof-of-concept prototype with the Spreadsheet Router that supports all features presented in Section 3. When using the prototype in practice, the editors demanded another feature that allows assigning process activities to a presentation tool (e.g., Microsoft PowerPoint). Such feature would enable them, for example, to easily create presentations for the management board. Altogether, the presented approach offers proper IT support of the riskbearing capacity procedure within a bank. Currently, we realize other parts of the risk reporting procedure as spreadsheet-driven processes. Finally, a limitation of the prototype is discussed. Therefore, recall the discussed case study (cf. Section 2) in which we revealed five spreadsheet-related drawbacks (SRD1-SRD5). The realized prototype provides features that address SRD1-SRD4. A feature that addresses SRD5, in turn, is currently not provided. Therefore, we work on this issue for the second version of the prototype.

\section{Related Work}

Three categories of related work need to be discussed in the context of this paper. First, there are approaches that apply process management technology to support activities for financial service providers. For example, [16] discusses 
performance issues of business processes in the context of ITIL processes, whereas [8] deals with the re-engineering of business processes in an international bank. Furthermore, there exist approaches that refer to financial scenarios in order to identify the characteristics of the underlying business processes [7]. Overall, the discussed approaches neither focus on human-centric, knowledge-intensive processes in the financial domain nor on the support of risk reporting procedures.

Second, approaches have to be considered that deal with office automation on one hand and spreadsheet-driven approaches on the other. Concerning the first category, such approaches already exist for quite some time. For example, in $[3,13]$ office automation approaches and their characteristics have been introduced. In particular, these approaches focus on the dynamic changes of office environments [4]. Furthermore, these approaches also discuss the need of workflows in the context of office automation and work distribution [9]. However, a tight integration of process management with spreadsheet technology is not presented in more detail by these approaches. Concerning the second category, many approaches exist in the context of information systems that apply spreadsheet-driven approaches to support the paradigm of end-user programming [1]. However, the use of spreadsheets as an advanced concept to perform process activities as we do is not considered so far.

Third, there are commercial tools that support spreadsheet-driven processes. Currently, the best match to this category constitutes the Microsoft Sharepoint Portal Server, which allows defining a workflow and assigning spreadsheet documents to its activities. The goal of such workflows is to easily exchange an document to coordinate procedures that particularly require the processing of numerical data. However, parts of an spreadsheet cannot be directly assigned to process activities with the standard features of the Sharepoint Portal Server.

\section{Summary and Outlook}

The paper presented an approach for the support of human-centric, knowledgeintensive business processes in the field of financial services. Regarding these processes, a variety of spreadsheet documents is used to cope with tasks and their respective processes in everyday business. However, existing IT solutions lack the combined support of spreadsheet technology with contemporary process management systems as required in the context of the present scenario. We propose a novel approach that exploits the benefits of using spreadsheets to perform process activities. Thereby, we illustrated the advantages of this approach in the context of risk reporting procedures. The latter are crucial for many financial service providers as regulatory requirements have been recently changed, but no proper IT solutions exist. We further showed how the approach can be applied to the risk-bearing capacity procedure as a fundamental pillar of risk reporting within a bank. Furthermore, we used the proof-of-concept prototype, which was realized on top of the Spreadsheet Router to execute the risk-bearing capacity procedure in terms of a spreadsheet-driven process. We consider the latter processes as crucial when targeting at the support of human-centric, knowledge- 
intensive business processes in the field of financial services. Altogether, with its spreadsheet-driven approach, the paper shows that the combination of process management with spreadsheet technology is promising.

\section{References}

1. Burnett, M., et al.: End-user software engineering with assertions in the spreadsheet paradigm. In: 25th Int'l Conf on Software Engineering. pp. 93-103. IEEE (2003)

2. Dadam, P., et al.: From ADEPT to AristaFlow BPM Suite: A Research Vision has become Reality. In: Int'l Conf on Business Process Management. pp. 529-531. Springer (2009)

3. Ellis, C., Nutt, G.: Office information systems and computer science. ACM Computing Surveys 12(1), 27-60 (1980)

4. Ellis, C., et al.: Dynamic Change within Workflow Systems. In: Proc of Conf on Organizational Computing Systems. pp. 10-21. ACM (1995)

5. Eppler, M., et al.: Improving Knowledge Intensive Processes through an Enterprise Knowledge Medium. In: Kommunikationsmanagement im Wandel, pp. 371-389. Springer (2008)

6. Garleanu, N., Pedersen, L.: Liquidity and Risk Management. Tech. rep., National Bureau of Economic Research (2007)

7. Korherr, B., List, B.: Extending the EPC and the BPMN with Business Process Goals and Performance Measures. In: ICEIS (3). pp. 287-294 (2007)

8. Küng, P., Hagen, C.: The fruits of Business Process Management: an experience report from a Swiss bank. Business Process Management Journal 13(4), 477-487 (2007)

9. Lapourtre, C., Rolf, G.: Distributed office automation system with specific task assignment among workstations (1992), US Patent 5,136,708

10. Mittnik, S.: Solvency ii calibrations: Where curiosity meets spuriosity. Munich: Center for Quantitative Risk Analysis (CEQURA), Department of Statistics, University of Munich (2011)

11. Mundbrod, N., Reichert, M.: Process-Aware Task Management Support for Knowledge-Intensive Business Processes: Findings, Challenges, Requirements. In: 3rd Int'l WS on Adaptive Case Management and other Non-workflow Approaches to BPM. IEEE Computer Society Press (2014)

12. Nicolas, M., Firzli, J.: A critique of the basel committee on banking supervision. Revue Analyse Financière 10 (2011)

13. Olson, M., Lucas Jr, H.: The impact of office automation on the organization: some implications for research and practice. Communications of the ACM 25(11), 838-847 (1982)

14. Reichert, M., Weber, B.: Enabling Flexibility in Process-Aware Information Systems: Challenges, Methods, Technologies (2012)

15. Schobel, J., et al.: A Mobile Service Engine Enabling Complex Data Collection Applications. In: Int'l Conf on Service-Oriented Computing. pp. 626-633 (2016)

16. Spremic, M., et al.: IT and Business Process Performance Management: Case Study of ITIL Implementation in Finance Service Industry. In: Proc of 30th Int'l Conf on Information Technology Interfaces. pp. 243-250. IEEE (2008) 